\title{
STATISTICS OF EVOLVING FAILURES IN ARRAYS OF PILLARS UNDER A MIXED-MODE LOAD TRANSFER
}

\author{
Tomasz Derda \\ Institute of Mathematics, Czestochowa University of Technology \\ Czestochowa, Poland \\ tomasz.derda@im.pcz.pl
}

\begin{abstract}
Progressively loaded multi-component systems may break when an initial sequence of failures of weakest components develops into an avalanche of failures that may involve all the system components. Once such an avalanche develops on each its stage, a load carried by components that are destroyed at this stage is transferred to remaining intact components. A different load transfer scenarios may be taken into account and used to model failures in overloaded systems. In this paper we apply a so-called Fibre Bundle Model to explore the effect of a mixed-mode load transfer in two-dimensional arrays of pillars. We use two types of loading processes: a quasi-static and sudden loading. By varying the weight parameter, we identify two distinct load transfer regimes: the global load sharing - when all intact components share the transferred load - and the local load sharing regime, when mainly the nearest neighbours suffer from the additional load. As a regime indicator, we use such characteristics like distribution of critical loads and function fitting probability of system breakdown.
\end{abstract}

Keywords: nanopillars, fibre bundle model, load transfer rule, probability and statistics

\section{Introduction}

Evolving failures in overloaded systems are complex phenomena and their modelling is important in many areas of science and engineering. Since fabrication processes inevitably introduce the structural heterogeneity of materials, then the failures of real materials usually cannot be described by linear models. Therefore, statistical models are widely used to study the fracture and breakdown processes. A Fibre Bundle Model (FBM) [1-3] is one of such statistical models. The FBM illustrates a stochastic fracture-failure process in disordered materials subjected to external load. The key aspect of this model is a load transfer rule which is responsible for the mechanism of redistribution of load carried by a destroyed element to other, still working elements. A set of possible load sharing rules can be classified according to ranges over which a given load transfer is achieved. As a consequence, two extreme rules are frequently applied within the FBM: global load sharing (GLS) and local load sharing (LLS). In the GLS rule, long-range interactions are assumed as all the intact elements equally share a load coming from broken elements. The LLS rule represents short-range interactions - the load from the destroyed element is redistributed only among its nearest intact neighbours. 
Since the GLS and the LLS rules are only rude approximations of real transfer processes, their mixture may then yield a better approximation to the reality. An original idea of a mixed-mode load sharing rule was given in [4] and applied to a simple one-dimensional system. The mixed-mode load redistribution scenario serves as an interpolation between two limiting cases, namely global and local load transfer. By varying the weight parameter, we identify two distinct load transfer regimes: the GLS - when all intact components share the transferred load - and the LLS regimes, when mainly nearest neighbours suffer from the additional load.

In this paper, we apply the mixed-mode FBM to damage processes in arrays of vertical pillars fixed on a flat substrate using two types of loading processes: a quasi-static and sudden loading.

\section{Model}

Our system is a set of $N$ pillars located in the nodes of the supporting lattice. We restrict ourselves to regular arrangements of pillars only i.e. we consider: hexagonal, square and triangular grids. However, the main interest is devoted to the square lattice case, which is seen as a set of $N=L \times L$ pillars with $L$ being the linear dimension. If it not specified otherwise, we explore the square lattice case.

Pillars, fabricated from alloys, are marked by their strength thresholds against an external load applied axially. Different alloys imperfections, as e.g. defects, influence the mechanical response of pillars under load. Hence, pillar-strength-thresholds $\sigma_{t h}^{i}, i=1,2, . ., N$ are represented by quenched random variables distributed according to the Weibull distribution

$$
P\left(\sigma_{t h}\right)=1-\exp \left\{-\left(\frac{\sigma_{t h}}{\lambda}\right)^{\rho}\right\}
$$

where $\rho$ is the shape parameter, also known as the Weibull index, and $\lambda$ is the scale parameter. The first parameter controls the amount of disorder in the system. In this work we assume $\lambda=1$ and $\rho=2$, which means strong disorder.

The system is subjected to an external load $F$ that is applied axially. In the following simulation it is instructive to consider two different loading procedures: quasi-static loading and sudden loading. For both of these processes the uniform loading is assumed. Nevertheless, the internal load transfers break homogeneities in loads distribution on individual pillars.

In the case of quasi-static loading, the external load $F$ is gradually increased up to the complete failure of the system. Initially the system is unloaded and intact. Then the load is uniformly increased on all the working pillars just to destroy the weakest one. Then, the increase of the external load is stopped and the load from the destroyed pillar is transferred to intact pillars. The load redistribution may lead to subsequent pillar failures that can provoke the next failures. A stable state 
is achieved if the final load redistribution does not trigger any failures. In such a situation, the external load has to be increased again. The above described dynamics is continued until destruction of all the pillars.

Sudden loading of the system is carried out by application of an external load $F$ which is kept constant during the entire loading process. Due to uniform loading, in the moment of application of load $F$ the load per pillar is $\sigma=F / N$, so all the pillars with strength thresholds smaller than $\sigma$ are immediately destroyed. Then, the load transfers may induce an avalanche of failures. This procedure leads to a stable state of the system which is either partially intact or fully destroyed. The third possibility is that the system is intact - only if $\sigma \leq \min \left(\left\{\sigma_{t h}^{i}\right\}\right)$.

As a load transfer rule, we apply mixed-mode load sharing with weight parameter $g$. In this scheme, when a pillar fails, fraction $g$ of its load is transferred locally and the rest ( $1-g$ fraction) is distributed globally. Therefore, the mixedmode load sharing is an interpolation mechanism between the GLS and the LLS rules, i.e. $g=0$ corresponds to the GLS rule and $g=1$ represents the LLS rule.

\section{Analysis of the simulation results}

Based on the model presented in the previous section, we have developed program codes for simulation of damage processes in the pillar arrays. Then, we have performed intensive computer simulations. Generally, we study the behaviour of the model from $g=0$ up to $g=1$ with a step of 0.05 . Because of computational time limitations, in some cases we increase the step to 0.1 .

\subsection{Quasi-static loading}

For the quasi-static loading, the damage process proceeds in an avalanche-like manner. Although initial load increases provoke only single pillar failures, further load increases involve bursts of failures. Such a single failure or a burst of pillar failures caused by load increase is called an avalanche $(\Delta)$. Application of the quasi-static loading process ensures obtaining the minimum external load $F$ that is needed to induce catastrophic avalanche $\Delta_{c}$ which contains all previously undestroyed pillars. Therefore, we can find the maximum value of applied load $F_{c}$ (total critical load) that can be supported by the system. The strength of the bundle can be scaled by the system size, thus giving critical load per pillar $\sigma_{c}=F_{c} / N$.

For the GLS rule, we assume that the support-pillar interface is perfectly rigid, whereas, in the case of the LLS rule, this support has a certain compliance. Thus, for the GLS, all the intact pillars are under equal load and the geometry of lattice is irrelevant. This is in contrast to the LLS rule, where load redistribution is localized and distribution of load is not homogeneous. By increasing $g$, load transfer 
changes from pure long-range $(g=0)$ to strictly localized $(g=1)$. In the following we analyse how it influences the mean value of critical load $\sigma_{c}$.

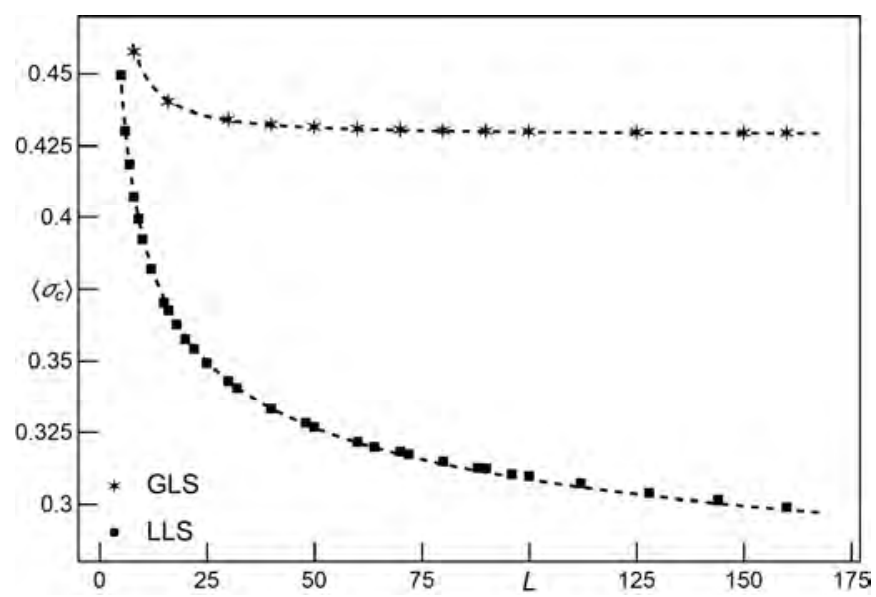

Fig. 1. Mean values of $\sigma_{c}$ for GLS and LLS. Dashed lines represent functions

(3) and (4)

It is known [5-7] that for the Weibull distribution of $\sigma_{t h}^{i}$ and the GLS rule, the mean critical load $\left\langle\sigma_{c}\right\rangle$ asymptotically tends to:

$$
\left\langle\sigma_{c}\right\rangle \underset{N \rightarrow \infty}{\longrightarrow} \rho^{-1 / \rho} e^{-1 / \rho}
$$

For finite $N$ a corrected formula holds:

$$
\left\langle\sigma_{c}\right\rangle=\rho^{-1 / \rho} e^{-1 / \rho}\left[1+0.996 N^{-2 / 3}\left(\frac{e^{2 / \rho}}{\rho}\right)^{1 / 3}\right] .
$$

Substantially different behaviour is detected for the LLS scheme, namely $\left\langle\sigma_{c}\right\rangle$ approaches zero in the asymptotic limit. We have found [8] that $\left\langle\sigma_{c}\right\rangle$ is well approximated by the following function:

$$
\left\langle\sigma_{c}\right\rangle=\frac{\beta}{(\ln N)^{\delta}} .
$$

Where $\beta=0.671$ and $\delta=0.35$ are fitted parameters. Formula (4) concerns 2D square lattice and was previously fitted to systems with uniformly distributed pillar-strength-thresholds [8]. 


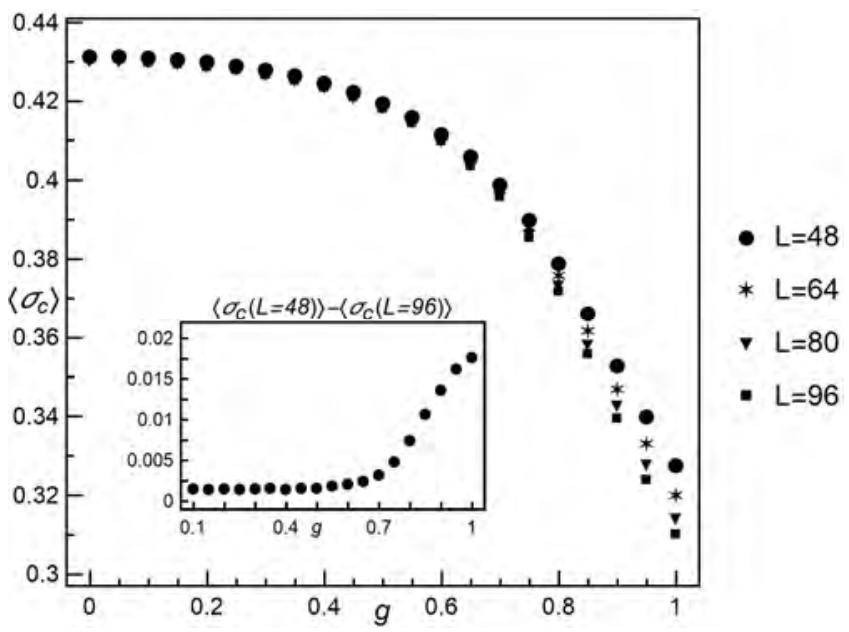

Fig. 2. The mean critical load versus weight parameter $g$ for different system sizes. The averages are taken from at least 5000 samples for each presented value. In the inset we show the results of subtracting $\left\langle\sigma_{c}\right\rangle$ for $L=48$ from $\left\langle\sigma_{c}\right\rangle$ for $L=96$

The mean critical load $\left\langle\sigma_{c}\right\rangle$ vs the weight parameter $g$ is presented in Figure 2 . In this Figure four different system sizes of arrays are represented. The function $\left\langle\sigma_{c}(g)\right\rangle$ monotonically decreases as $g$ increases. This means that load transfer becomes more and more localised and this localization causes weakening of the system. It is clearly seen that if $g \leq 0.65$ then the computed values of $\left\langle\sigma_{c}\right\rangle$ are practically identical regardless the system size. From this fact we can conclude that long-range interactions dominate. For all studied arrays when values of $g$ were greater than 0.65 the differences between $\left\langle\sigma_{c}\right\rangle$ were visibly stronger (see inset in Fig. 1). Starting from $g=0.8$ we distinctly observe size-dependent behaviour and this value of $g$ marks a dominance of the LLS regime. Very similar behaviour was published in [4]. However, the results of our work and the results from [4] concern a different system's dimension and also strength-thresholds are differently distributed.

To analyse the impact of pillar arrangements on arrays' behaviour, we compare $\left\langle\sigma_{c}\right\rangle$ for three regular lattices (see Fig. 3). The lattices are characterised by coordination numbers $z$. Namely, hexagonal: $z=3$, square: $z=4$ and triangular: $z=6$. In the classical LLS scheme, as the number of nearest neighbours increases the load from the destroyed pillar becomes more dispersed and thus leads to a strengthening of the system. For the LLS, the hexagonal system is the weakest, while the triangular system is the strongest one. The same behaviour is observed for the mixed-mode $(g>0)$ although, for smaller values of $g$, the differences are close to 0 . From $g=0.5$, the results for hexagonal lattice start to visibly differ from 
the results obtained for square and triangular geometries, while the results for these two geometries are almost equal up to $g=0.8$.

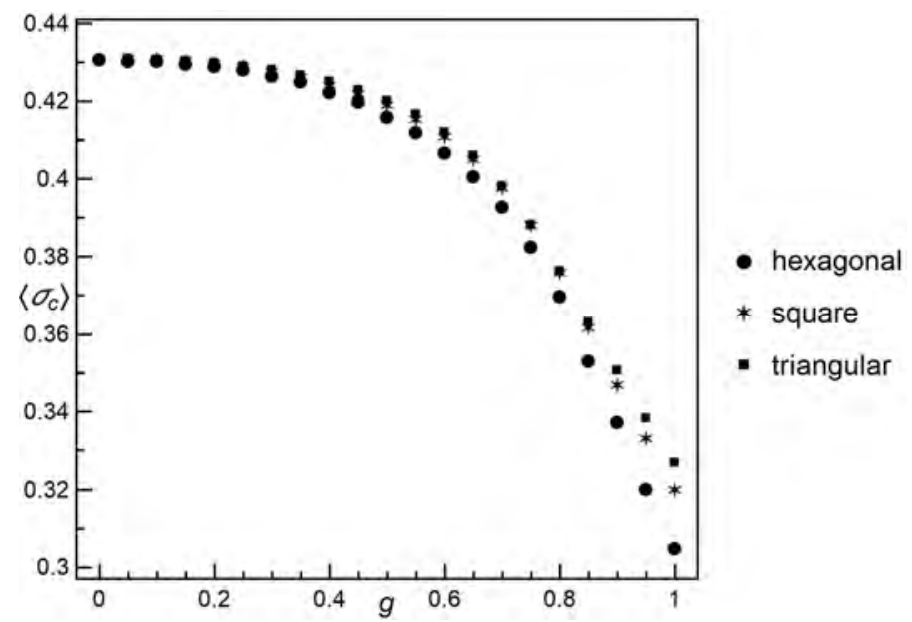

Fig. 3. The mean critical load as a function of weight parameter $g$ for different system geometries and $N=64^{2}$. The averages are taken from at least 5000 samples for each presented value

In our previous works, we have noticed that for the LLS model, the distribution of $\sigma_{c}$ can be fitted by three-parameter skew normal distribution (SND) with probability density function $[9,10]$ :

$$
p\left(\sigma_{c}\right)=\frac{\exp \left[-\frac{\left(\sigma_{c}-\xi\right)^{2}}{2 \omega^{2}}\right] \operatorname{erfc}\left[-\frac{\alpha\left(\sigma_{c}-\xi\right)}{\sqrt{2} \omega}\right]}{\sqrt{2 \pi} \omega}
$$

and cumulative distribution function (CDF)

$$
P\left(\sigma_{c}\right)=\frac{1}{2} \operatorname{erfc}\left(-\frac{\sigma_{c}-\xi}{\sqrt{2} \omega}\right)-2 \mathrm{~T}\left(\frac{\sigma_{c}-\xi}{\omega}, \alpha\right)
$$

where $\xi, \omega, \alpha$ are location, scale and shape parameters, respectively. Function $\operatorname{erfc}(z)$ represents complimentary error function

$$
\operatorname{erfc}(z)=1-\operatorname{erf}(z)=\frac{2}{\sqrt{\pi}} \int_{0}^{z} \exp \left(-t^{2}\right) d t
$$

and $\mathrm{T}(x, a)$ is Owen's T function: 


$$
\mathrm{T}(x, a)=(2 \pi)^{-1} \int_{0}^{a} \frac{\exp \left[-\frac{1}{2} x^{2}\left(1+t^{2}\right)\right]}{\left(1+t^{2}\right)} d t \quad(-\infty<x, a<+\infty)
$$

In the case of the GLS model, $\sigma_{c}$ is normally distributed. Skew normal distribution generalizes the normal distribution by introducing a non-zero skewness. Therefore, we have fitted distribution of $\sigma_{c}$ for the mixed-mode scheme by skew normal distribution. But first, in Figure 4, we present results of skewness of $\sigma_{c}$ distribution for different values of $g$ and chosen system sizes. We can see that up to $g=0.7$ distribution of $\sigma_{c}$ is approximately symmetric. From $g=0.7$ up to $g=1$ skewness is negative like in the LLS case and moderate skewness is approached.

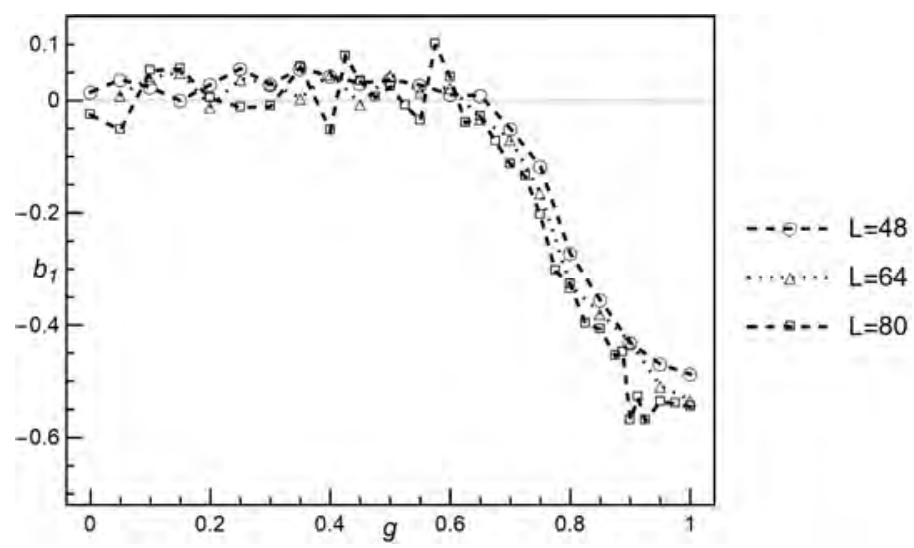

Fig. 4. The skewness of critical load as a function of weight parameter $g$ for different system sizes. The results are taken from at least 5000 samples for each presented value

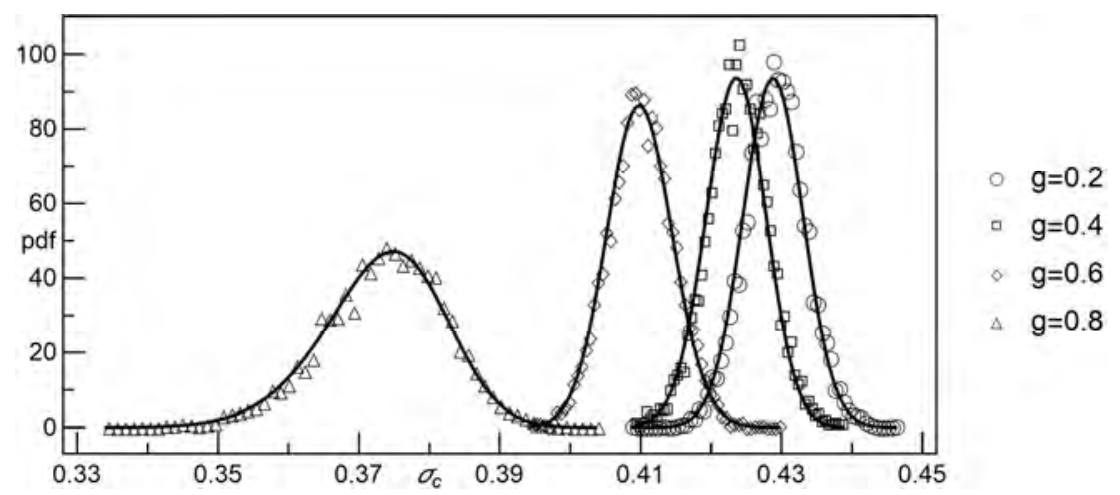

Fig. 5. Empirical probability density functions of the $\sigma_{c}$ in an array of $80 \times 80$ pillars. The solid lines represent a probability density function of skew normally distributed $\sigma_{c}$ with parameters computed from the samples 


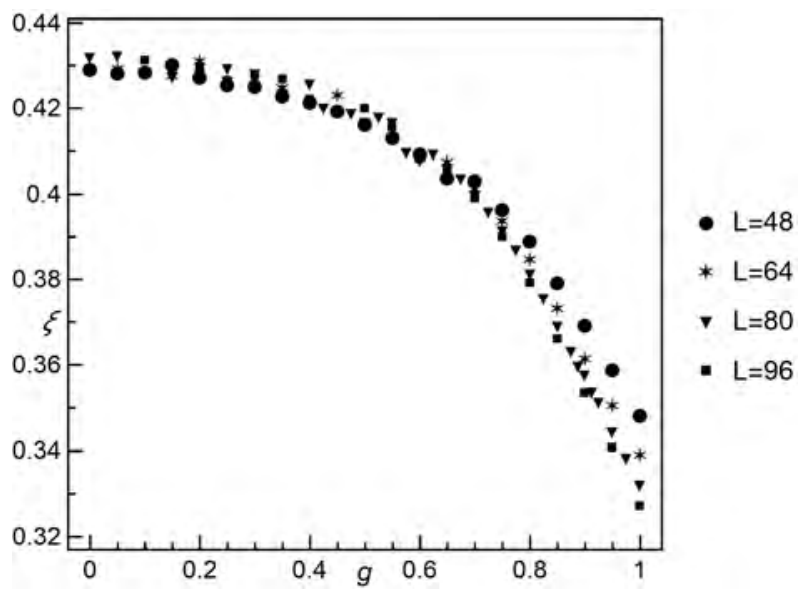

Fig. 6. The location parameter $\xi$ of skew normal distribution as a function of $g$ for different system sizes

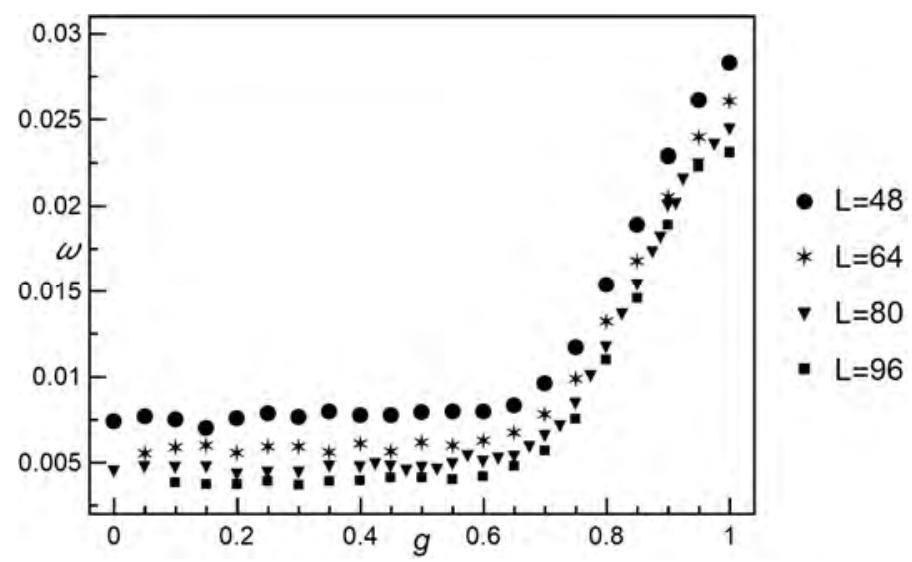

Fig. 7. The scale parameter $\omega$ of skew normal distribution as a function of $g$ for different system sizes

Figure 5 illustrates exemplary empirical probability density functions of $\sigma_{c}$ for different values of weight parameter $g$. Figures 6-8 graphically report fitted values of parameters of skew normally distributed $\sigma_{c}$. Each presented result is based on at least 20,000 independent samples $(L=48), 10,000$ samples $(L=64)$ and 5,000 samples $(L=80$ and $L=96$ ). In Figure 5, two regimes can be noticed. For $g \leq 0.6$ we see three curves similar to each other in terms of dispersion, which is low in comparison to dispersion for $g=0.8$. This observation is supported by Figure 7, where a noticeable increase of estimated scale parameter $\omega$ is observed 
from $g=0.7$ up to $g=1$, whereas up to $g=0.6$ fitted values of $\omega$ are almost constant. Figure 8 depicts estimated values of shape parameter $\alpha$. Up to $g=0.65$ values of $\alpha$ are scattered around zero in the range of approximately $(-1,1)$. From $g=0.7$ up to $g=1$, values of $\alpha$ generally decrease and all are negative. It thus allows us to clearly differentiate between two regimes. We have also tested a hypothesis about normal distribution of $\sigma_{c}$ (significance level of 0.05 ). The hypothesis was not rejected up to $g=0.7$ for all analysed system sizes. From $g=0.75$ up to $g=1$, all cases were rejected.

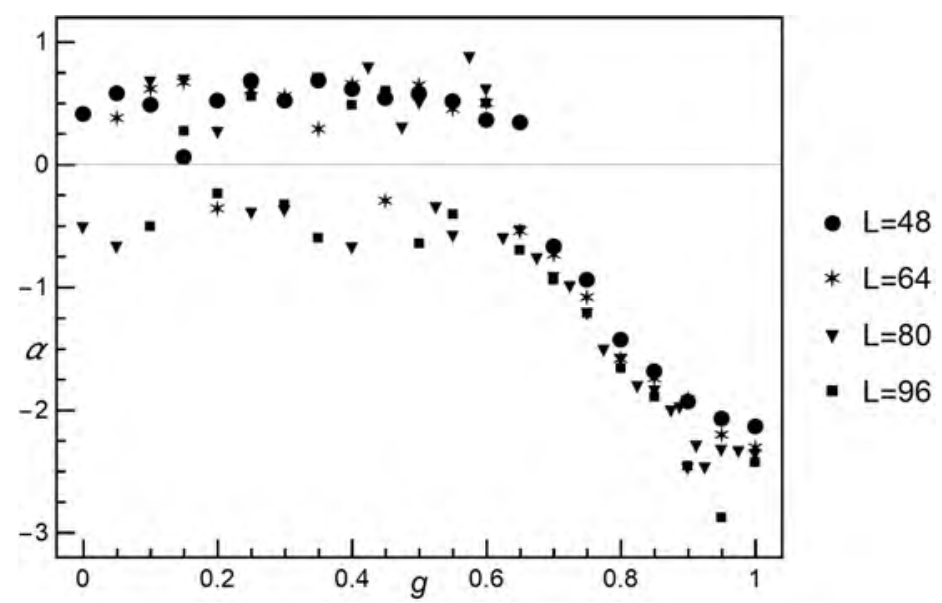

Fig. 8. The shape parameter $\alpha$ of skew normal distribution as a function of $g$ for different system sizes

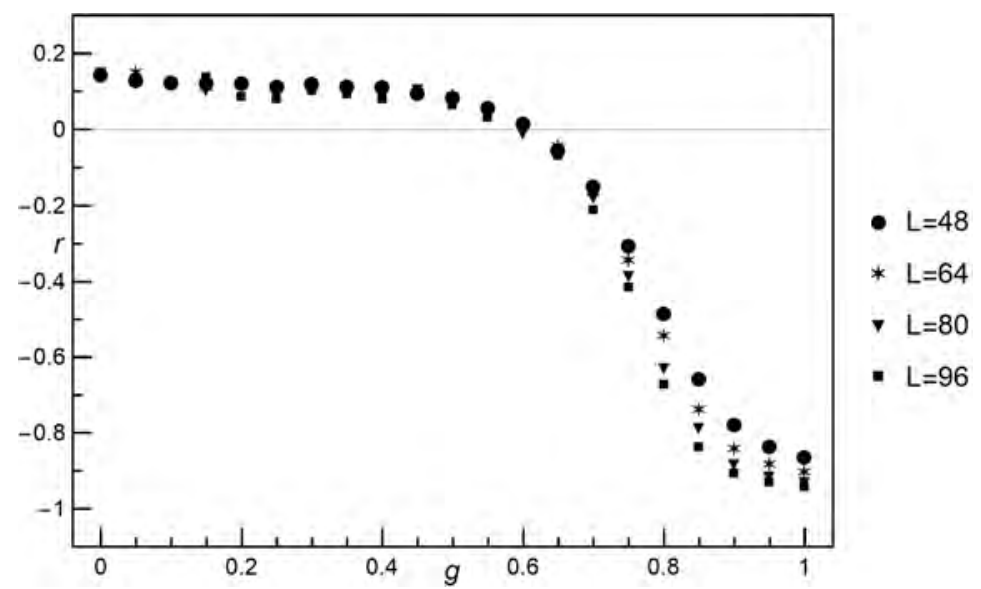

Fig. 9. The correlation coefficient $r$ between $\sigma_{c}$ and $\Delta_{c}$ as a function of $g$ for different system sizes 
The last analysed quantity in this subsection is Pearson correlation coefficient $r$ between critical load $\sigma_{c}$ and size of the catastrophic avalanche $\Delta_{c}$. In the work [11] we have reported a very strong negative linear relationship for the LLS rule and no or very weak correlation for the GLS rule. In the mixed-mode model (see Fig. 9) no or very weak correlation is up to $g=0.7 \quad(|r| \leq 0.2)$. Then, as $g$ increases, the correlation becomes stronger and stronger, thus showing the transition from GLS regime to LLS regime. From $g=0.75$ up to $g=1$ we observe that correlation is ordered according to system size that indicates size effect, which is characteristic for the short-range interactions.

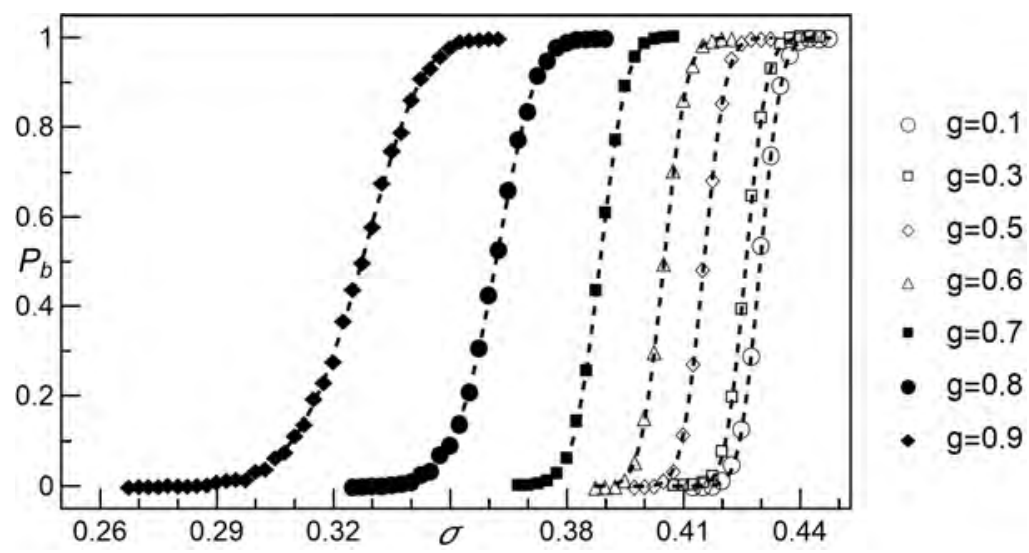

Fig. 10. Empirical breakdown probability $P_{b}$ as a function of initial load per pillar $\sigma$ for different values of weight parameter $g$. All presented data are calculated from 2000 statistically independent samples. System size $N=80 \times 80$. The dashed lines represent function (6) with parameters computed from simulations

\subsection{Sudden loading}

In the beginning of this subsection, we analyse probabilities of breakdown $\left(P_{b}\right)$ of systems loaded by finite force $F$. Application of $\sigma=F / N$ allows us to compare results for different system sizes. Figure 10 depicts empirical breakdown probabilities for chosen values of weight parameter $g$. It is seen that fitted curves are ordered according to $g$. In addition, the distance (in the $x$-direction) between consecutive curves seems to increase as $g$ is increased. For $g \leq 0.7$ the fitted 
curves sharply increase, whereas for $g=0.8$ and $g=0.9$, values of $P_{b}$ increase more slowly. A resulting three-dimensional plot of breakdown probability in the function of $g$ and $\sigma$ is shown in Figure 11 .

For fitting our data we employ cumulative distribution function of skew normal distribution, and thus we rewrite formula (6):

$$
P_{b}(\sigma)=\frac{1}{2} \operatorname{erfc}\left(-\frac{\sigma-\xi_{S L}}{\sqrt{2} \omega_{S L}}\right)-2 \mathrm{~T}\left(\frac{\sigma-\xi_{S L}}{\omega_{S L}}, \alpha_{S L}\right)
$$

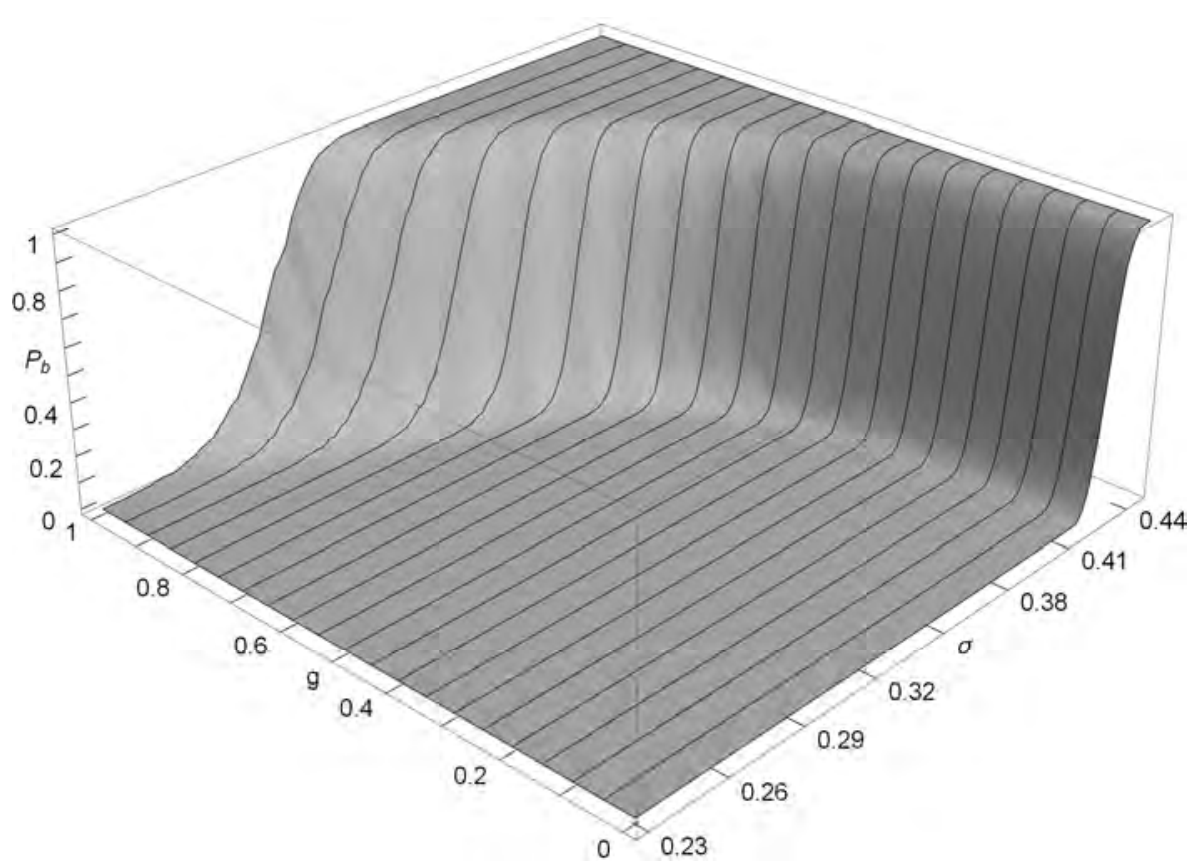

Fig. 11. Breakdown probability $P_{b}$ as a function of the two variables $\sigma$ and $g$.

System size $N=80 \times 80$

Figures 12-14 show fitted values of parameters $\xi_{S L}, \omega_{S L}$ and $\alpha_{S L}$ for different system sizes. It can be noticed that the behaviour of these parameters is very similar to the behaviour of their counterparts in the case of quasi-static loading. This evidences that the two applied loading procedures are equivalent. 


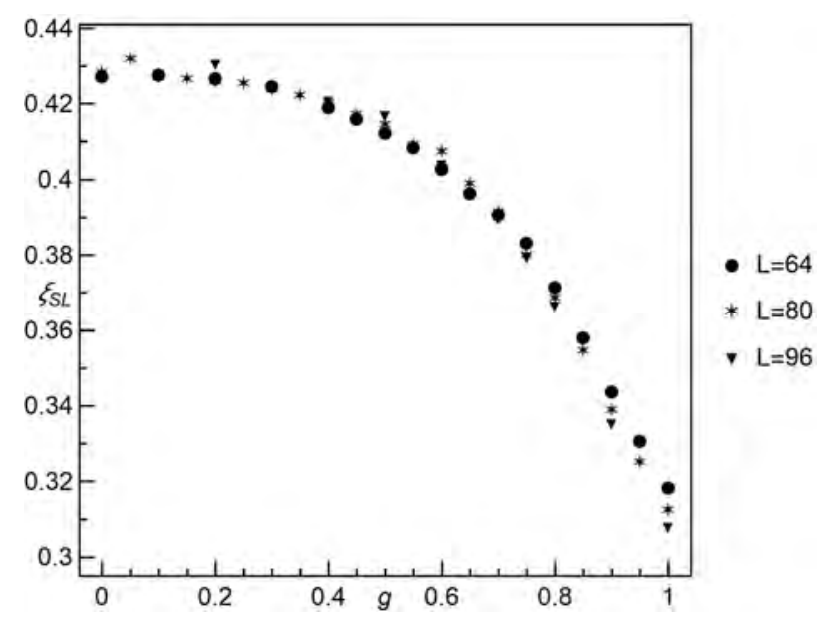

Fig. 12. The parameter $\xi_{S L}$ of formula (9) as a function of $g$ for different system sizes

The dominance of short range interactions is distinctly visible for $g>0.7$ (see Figs. 13 and 14). The GLS regime dominates up to $g=0.65$.

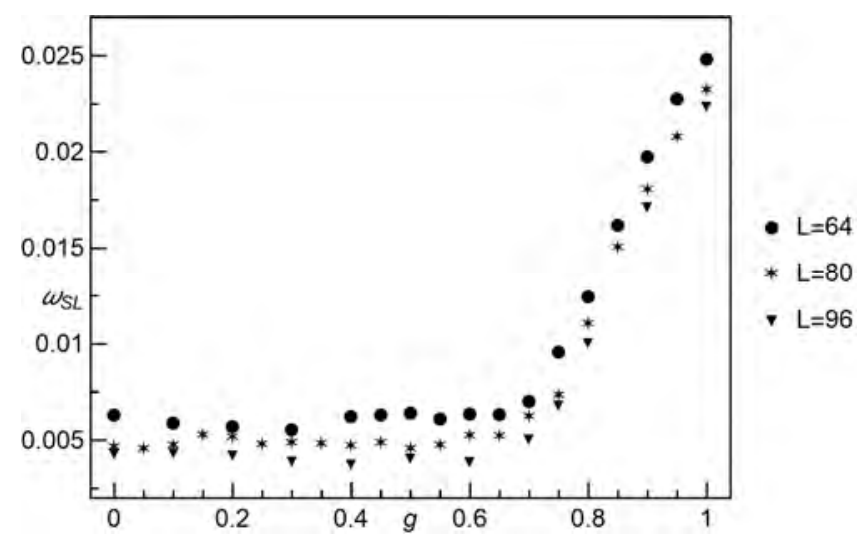

Fig. 13. The parameter $\omega_{S L}$ of formula (9) as a function of $g$ for different system sizes

At the beginning of the section, we have mentioned the distances between curves for consecutive values of $g$. A quartile can serve as a tool to measure the distance in the $x$-direction. Using values of parameters $\xi_{S L}, \omega_{S L}$ and $\alpha_{S L}$ we compute quartiles of the skew normal distribution. Then we calculate differences between quartiles for $g-0.05$ and for $g$. By that means, we obtain distances between consecutive curves with a step of 0.05 . The results are plotted in Figure 15. It is seen that the distance between consecutive curves is an increasing function up to $g=0.9$, then the distance starts to decrease. This behaviour shows that for $g=0.9$ short range interactions in the system are so prevalent that a further increase of $g$ is less significant. 


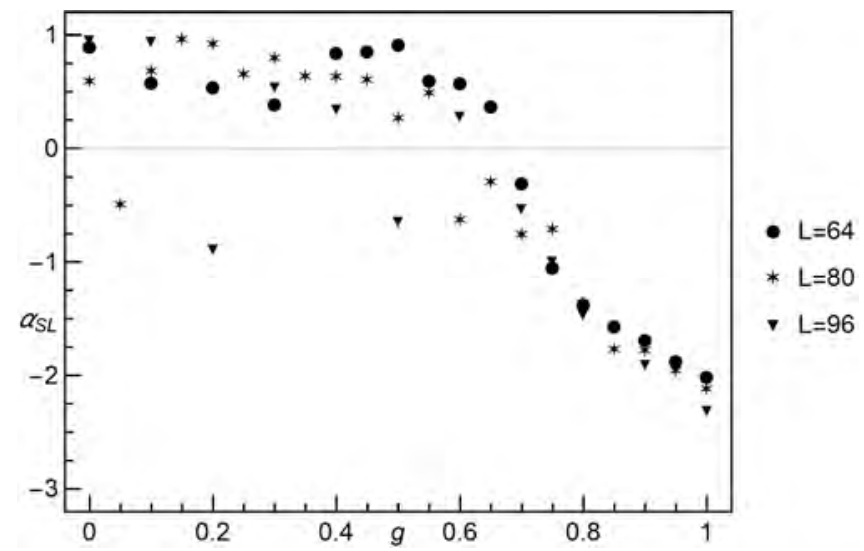

Fig. 14. The parameter $\alpha_{S L}$ of formula (9) as a function of $g$ for different system sizes

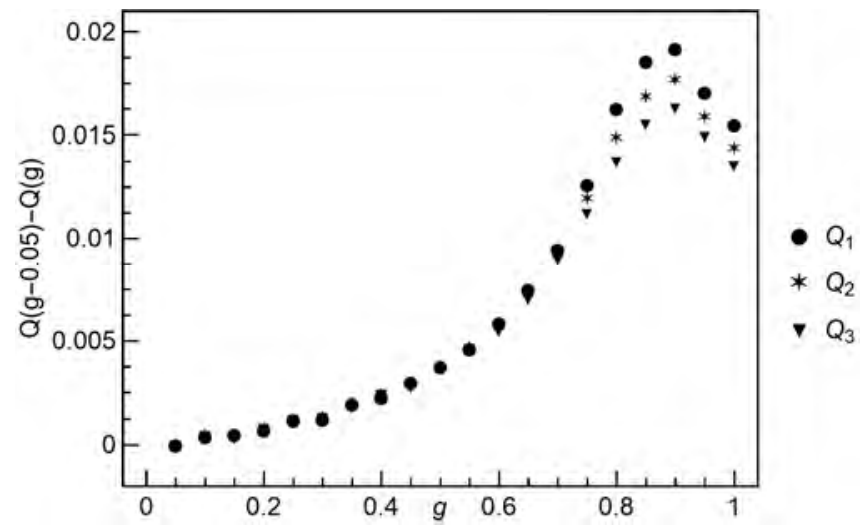

Fig. 15. The results of subtracting quartile of SND for $g-0.05$ from quartile of SND for $g$. Parameters of SND are based on the simulation results. The results concern systems with $N=80 \times 80$ pillars

In addition to the study of $P_{b}$, it is also important to analyse fraction $(U)$ of pillars that remain intact under a given load $\sigma$. Figure 16 illustrates mean values of $U$ as a function of $\sigma$. The ordering of curves joining points remains the same as in the $P_{b}$ case. For small and moderate values of $g$, the curves decrease slowly and then, from a certain point, they begin to decline rapidly. This is in contrast to greater values of $g$, where the curves decrease significantly slower. Distinction of the system regime can be done by comparing appropriate curves for different system sizes. For the GLS system, we have observed that curves are crossing each other, whereas for the LLS rule the curves diverge without crossing themselves. In our case, the intersection of the curves persists up to $g=0.7$. This suggests that for these values of $g$ long range interactions are dominating. However, starting from $g=0.8$ a LLS-like behaviour is observed (see Fig. 17). 


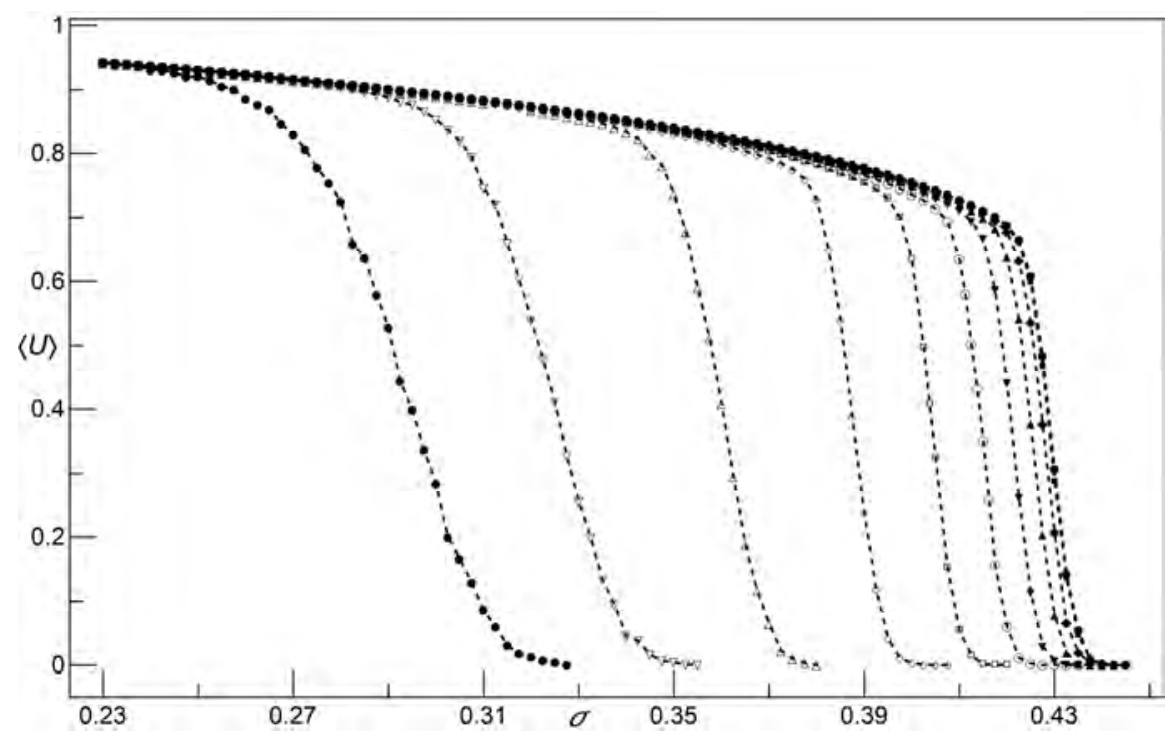

Fig. 16. Mean fraction of intact pillars $\langle U\rangle$ vs load per pillar $\sigma$. System size $N=96 \times 96$. Values of $g$ are from 0 to 1 with step of 0.1 (from right to left)

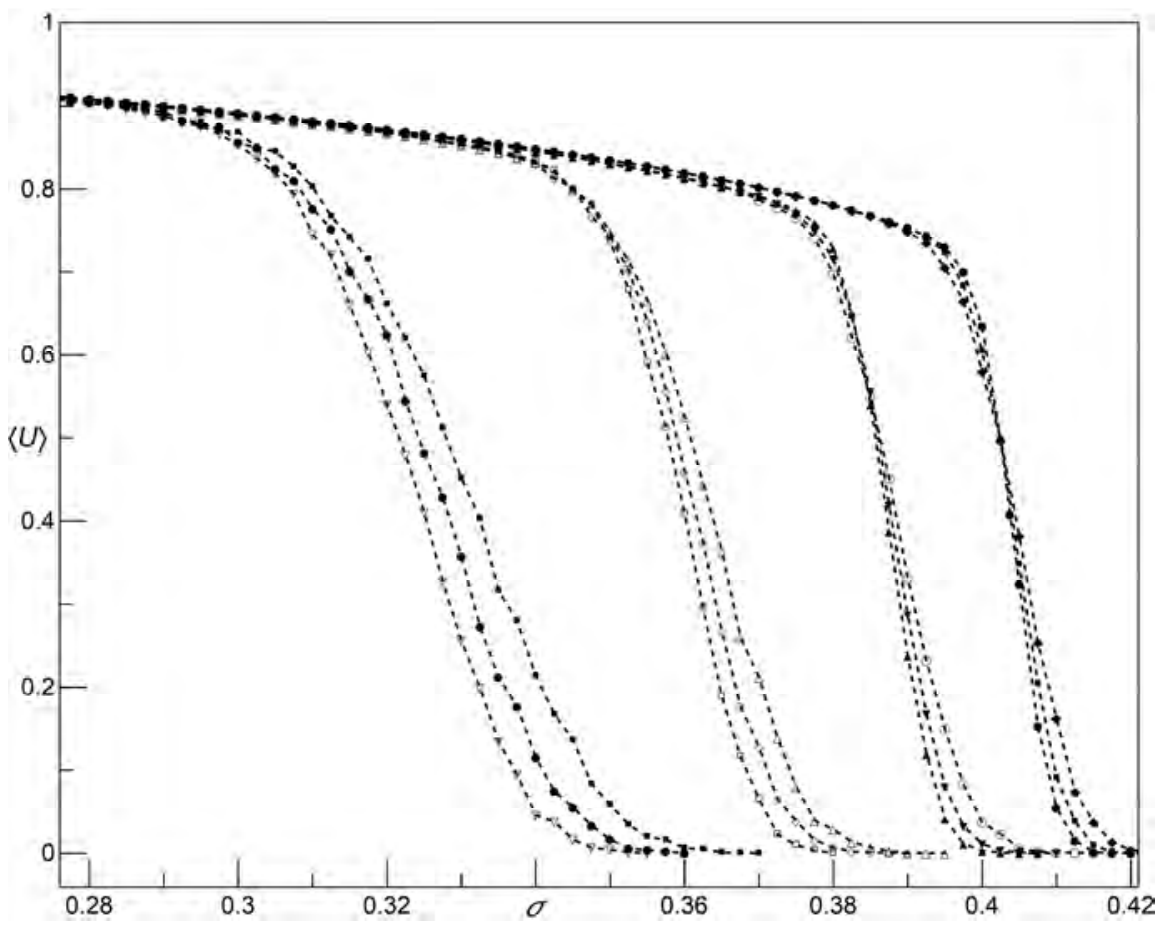

Fig. 17. Mean fraction of intact pillars $\langle U\rangle$ vs load per pillar $\sigma$. The curves are grouped according to $g=0.6,0.7,0.8,0.9$ (right to left). Each group of curves is composed from results for three system sizes $L=64,80,96$ 
To analyse the system regime we also use the reduced fourth-order Binder cumulant $[12,13]$

$$
B=1-\frac{\left\langle U^{4}\right\rangle}{3\left\langle U^{2}\right\rangle^{2}}
$$

Using $B$, in Figures 18 and 19, we have plotted examples of both the GLS and LLS regimes. Figure 18 presents cases with the GLS dominance. The curves joining points are crossing each other. However, density of the points is too small to accurately determine the crossing points. Examples of the short-range dominance with no crossing points is presented in Figure 19.

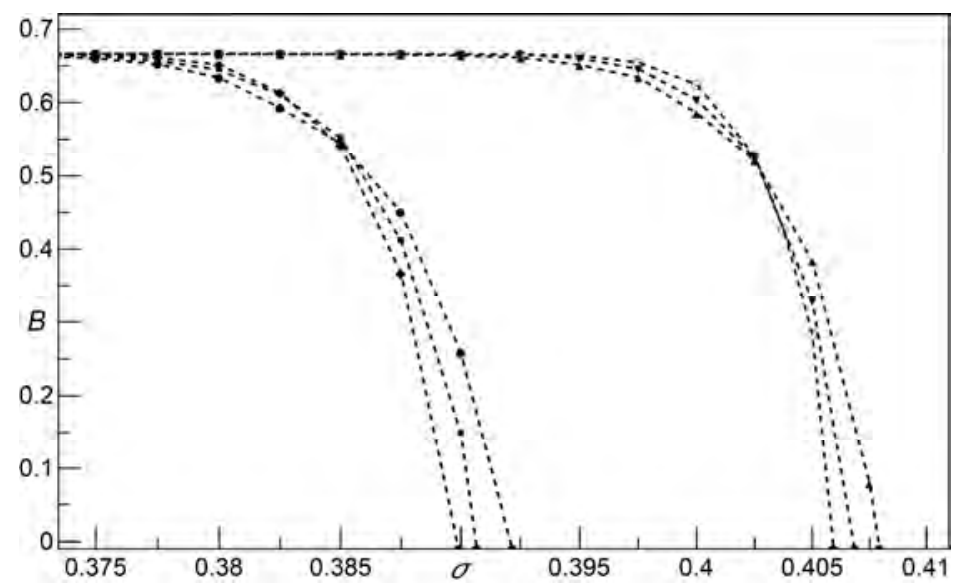

Fig. 18. The Binder cumulant $B$ vs load per pillar $\sigma$ - example of the GLS behaviour.

Two groups of curves are plotted: $g=0.6$ (right), $g=0.7$ (left). Each group of curves represents results for $L=64,80,96$

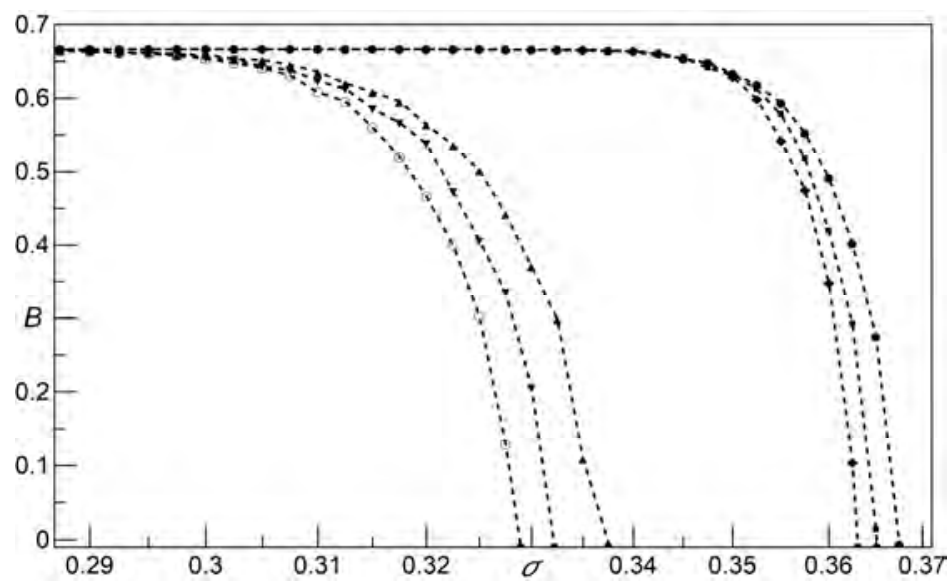

Fig. 19. The Binder cumulant $B$ vs load per pillar $\sigma$ - example of the LLS behaviour.

Two groups of curves are plotted: $g=0.8$ (right), $g=0.9$ (left). Each group of curves represents results for $L=64,80,96$ 
Finally, we compare approximations of $P_{b}$ by cumulative distribution functions of two distributions, namely normal and skew normal. To study the quality of approximation we apply the mean absolute error (MAE). The results of MAE are reported in Figure 20. Up to $g=0.65$, both of the functions generate almost equal errors (GLS regime), then mean absolute errors for normal distribution are greater than their skew-normal counterparts (LLS behaviour). From $g=0.8$ the difference is becoming considerable and thus suggests distinct LLS regime.

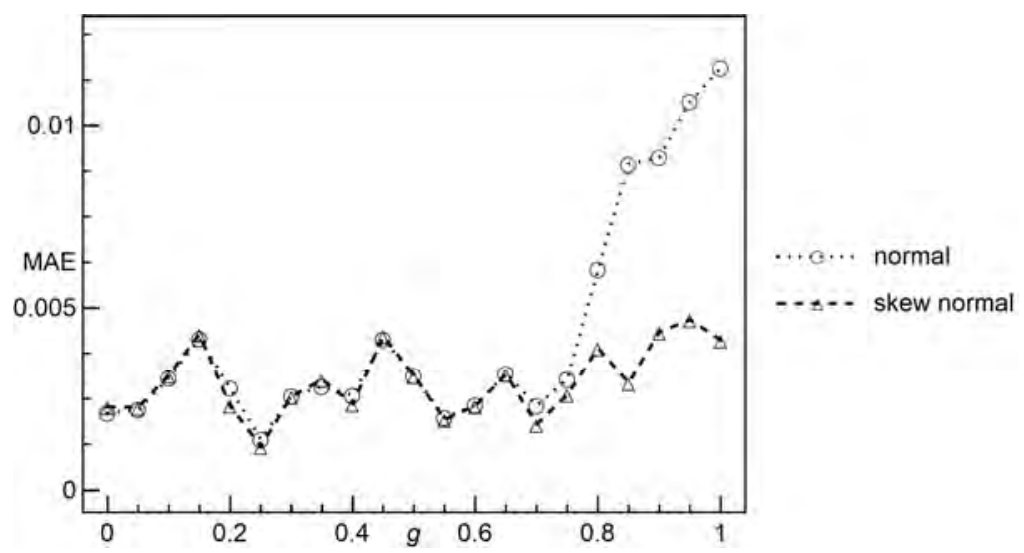

Fig. 20. Mean absolute errors of $P_{b}$ approximation using: CDF of the SND and CDF of the normal distribution. The results concern systems with $N=80 \times 80$ pillars

\section{Conclusions}

By means of numerical simulation, we have studied breakdown processes in the mixed-mode load transfer model of nanopillar arrays subjected to external load. This model is a complete GLS scheme for weight parameter $g=0$ and pure LLS scheme for $g=1$.

Application of two different loading procedures allowed us to analyse two quantities i.e. critical load and breakdown probability. We have shown that distribution of critical load can be nicely fitted by the skew normal distribution, and breakdown probability is well approximated by the cumulative distribution function of this distribution. The parameters of these functions can serve as the indicators of the system regime. We have tuned values of $g$ from 0 to 1 with step of 0.05 . We have observed that up to $g=0.65$ long range interactions prevail (GLS behaviour), whereas from $g=0.8$ we see distinct LLS behaviour with short range interactions. Between the two mentioned above values of $g$, the crossover regime is present. Similar conclusions can be obtained by analysing fraction $U$ of intact pillars under given load and values of Binder cumulant calculated for $U$. 


\section{References}

[1] Hansen A., Hemmer P.C., Pradhan S., The Fiber Bundle Model: Modeling Failure in Materials, Wiley 2015.

[2] Alava M.J., Nukala P.K.V.V., Zapperi S., Statistical models of fracture, Adv. In Physics 2006, 55, 349-476.

[3] Pradhan S., Hansen A., Chakrabarti B.K., Failure processes in elastic fiber bundles, Rev. Mod. Phys. 2010, 82, 499-555.

[4] Pradhan S., Chakrabarti B.K., Hansen A., Crossover behavior in a mixed-mode fiber bundle model, Phys. Rev. E 2005, 71, 036149.

[5] McCartney L.N., Smith R.L., Statistical Theory of the Strength of Fiber Bundles, J. Appl. Mech. 1983, 50(3), 601-608.

[6] Smith R.L., The asymptotic distribution of the strength of a series-parallel system with equal load-sharing, The Annals of Probability 1982, 10(1), 137-171.

[7] Porwal P.K., Beyerlein I.J., Phoenix S.L., Statistical strength of a twisted fiber bundle: an extension of Daniels equal-load-sharing parallel bundle theory, Journal of Mechanics of Materials and Structures 2006, 1(8), 1425-1447.

[8] Domański Z., Derda T., Sczygiol N., Critical avalanches in fiber bundle models of arrays of nanopillars, Proceedings of the International MultiConference of Engineers and Computer Scientists 2013, Vol. II, IMECS 2013, March 13-15, 2013.

[9] Derda T., Stochastic local load redistribution in the fibre bundle model of nanopillar arrays, J. Appl. Math. Comput. Mech. 2015, 14(4), 19-30.

[10] Domański Z., Derda T., Distributions of critical load in arrays of nanopillars, Proceedings of the World Congress on Engineering (WCE 2017), Vol. II, 797-801.

[11] Derda T., Avalanche statistics in transfer load models of evolving damage, Scientific Research of the Institute of Mathematics and Computer Science 2011, 10(1), 21-31.

[12] Binder K., Heermann D., Monte Carlo Simulations in Statistical Physics, Springer, Berlin 1988.

[13] Biswas S., Chakrabarti B.K., Crossover behaviors in one and two dimensional heterogeneous load sharing fiber bundle models, Eur. Phys. J. B 2013, 86, 160. 